\title{
Critical Phenomena of Charged AdS Black Holes in Rastall Gravity
}

\author{
De-Cheng Zou $\mathbb{D}^{1}{ }^{1}$ Ming Zhang, ${ }^{2}$ Chao Wu, ${ }^{1}$ and Rui-Hong Yue ${ }^{1}$ \\ ${ }^{1}$ Center for Gravitation and Cosmology, College of Physical Science and Technology, Yangzhou University, Yangzhou 225009, China \\ ${ }^{2}$ Faculty of Science, Xian Aeronautical University, Xian 710077, China
}

Correspondence should be addressed to De-Cheng Zou; dczou@yzu.edu.cn

Received 2 September 2019; Revised 27 September 2019; Accepted 28 September 2019; Published 24 January 2020

Academic Editor: Sunny Vagnozzi

Copyright ( 2020 De-Cheng Zou et al. This is an open access article distributed under the Creative Commons Attribution License, which permits unrestricted use, distribution, and reproduction in any medium, provided the original work is properly cited.

\begin{abstract}
We construct analytical charged anti-de Sitter (AdS) black holes surrounded by perfect fluids in four dimensional Rastall gravity Then, we discuss the thermodynamics and phase transitions of charged AdS black holes immersed in regular matter like dust and radiation, or exotic matter like quintessence, $\Lambda$ CDM type, and phantom fields. Surrounded by phantom field, the charged AdS black hole demonstrates a new phenomenon of reentrant phase transition (RPT) when the parameters $Q, N_{p}$, and $\psi$ satisfy some certain condition, along with the usual small/large black hole $(\mathrm{SBH} / \mathrm{LBH})$ phase transition for the surrounding dust, radiation, quintessence, and cosmological constant fields.
\end{abstract}

\section{Introduction}

As well known, Einstein's theory of general relativity (GR) has made a lot of interesting predictions for solar system experiments, which are in perfect agreement with the observational data. On larger (cosmological) scales, there are lines of strong observational evidence implying that dark matter (DM) and dark energy (DE) account for about $95 \%$ of the universe $[1,2]$. From these observations, many theoretical models have been presented in Refs. [3-5]. In 1972, one of the potential gravity theories was proposed by Rastall [6, 7]. In the Rastall gravity theory, the usual conservation law of the energymomentum tensor $\left(T_{; \mu}^{\mu v}=0\right)$ is not obeyed, and the energymomentum tensor satisfies

$$
T_{; \mu}^{\mu \nu}=\lambda R^{v},
$$

where $R$ is the Ricci scalar, and the parameter $\lambda$ measures the potential deviations of Rastall theory from GR. It is interesting that all electrovacuum solutions in GR are also solutions of Rastall gravity. However, if matter field (nonvanishing trace) is introduced, the spacetime depends on the Rastall parameter $\lambda$. As the simplest modified gravity scenario, it provides an explanation of the inflation problem, and realizes the late-time acceleration and other cosmological problems [8-11]. Until now, many works on the various black hole solutions have been also investigated in Rastall theory. The spherically symmetric black hole solutions were constructed in Refs. [12-16], the rotating black holes in Refs. $[17,18]$, the thermodynamics of black holes in Refs. [19-22], and also instability of black holes in Refs. [23, 24].

According to the AdS/CFT correspondence, the bulk AdS black hole spacetimes admit a gauge duality description by thermal conformal field theory living on the AdS boundary [25-27]. For example, the usual Hawking-Page phase transition [28] in four dimensional GR can be interpreted as a confinement/deconfinement phase transition in the dual quark gluon plasma [29]. Recently, the study of thermodynamics of AdS black holes has been extended to the extended phase space, where the cosmological constant is treated as the pressure of the black hole $[30,31]$. In the extended phase space, charged AdS black hole admits a more direct and precise coincidence between the first order small/large black holes (SBH/ LBH) phase transition and Van der Waals (VdW) liquid-gas phase transition [32], and both systems share the same critical exponents near the critical point. More discussions in this direction can be found as well in Refs. [33-61], including reentrant phase transitions and some other phase transitions. In the Rastall theory, Ali recovered the existence of VdW like 
TABLE 1: The condition for $\rho_{s} \geq 0$.

\begin{tabular}{llll}
\hline$N_{s}>0 \cup W_{s}<0$ & $\omega_{s}>-1 \cup-2<\xi<1$ & $\omega_{s}<-1 \cup \xi>1$ & $\omega_{s}<-1 \cup \xi<-2$ \\
$N_{s}<0 \cup W_{s}>0$ & $\omega_{s}<-1 \cup-2<\xi<1$ & $\omega_{s}>-1 \cup \xi>1$ & $\omega_{s}>-1 \cup \xi<-2$ \\
\hline
\end{tabular}

SBH/LBH phase transition for AdS black holes in four dimensional spacetimes [62]. Motivated by this result, it is interesting to explore the effect of charge $Q$ on the critical behaviors for charged AdS black holes in four dimensional Rastall theory. We hope to reveal a more fascinating phenomenon.

This paper is organized as follows. In Section 2, the analytical solution for static spherical symmetric charged AdS black holes are obtained in four dimensional Rastall theory. In Section 3, we study the critical behaviors of the charged AdS black holes in context of $P-V$ criticality and phase diagrams. We end the paper with conclusion and discussion in Section 4.

\section{Charged AdS Black Hole Solutions in Rastall Theory}

Considering Equation (1), the field equation including the negative cosmological constant $\Lambda$ can be written as [62]

$$
G_{\mu \nu}+\Lambda g_{\mu \nu}+\kappa \lambda g_{\mu \nu} R=T_{\mu \nu},
$$

where $\kappa$ equals to $8 \pi G_{N}$ and $G_{N}$ is the Newton gravitational coupling constant.

In order to derive the black hole solutions, we consider the general spherical symmetric spacetime metric

$$
d s^{2}=-f(r) d t^{2}+\frac{1}{f(r)} d r^{2}+r^{2}\left(d \theta^{2}+\sin ^{2} \theta d \vartheta^{2}\right),
$$

where $f(r)$ is a generic metric function depending on the radial coordinate $r$. Using this metric, the nonvanishing components of right hand side of Equation (2) are

$$
\begin{aligned}
& H_{0}^{0}=H_{1}^{1}=G_{0}^{0}+\Lambda+\kappa \lambda R=\frac{1}{r^{2}}\left(f^{\prime} r-1+f\right)+\Lambda+\kappa \lambda R, \\
& H_{2}^{2}=H_{3}^{3}=G_{2}^{2}+\Lambda+\kappa \lambda R=\frac{1}{r^{2}}\left(r f^{\prime}+\frac{1}{2} r^{2} f^{\prime \prime}\right)+\Lambda+\kappa \lambda R,
\end{aligned}
$$

with the Ricci scalar $R=-\left(1 / r^{2}\right)\left(r^{2} f^{\prime \prime}+4 r f^{\prime}-2+2 f\right)$.

On the other hand, the total energy-momentum tensor $T_{\mu \nu}$ supporting this spacetime takes the following form $[13,14]$

$$
T^{\mu}{ }_{v}=E^{\mu}{ }_{v}+\mathcal{T}^{\mu}{ }_{v}
$$

where $E_{v}^{\mu}$ is given by

$$
E_{\mu \nu}=\frac{2}{\kappa}\left(F_{\mu \alpha} F_{v}^{\alpha}-\frac{1}{4} g_{\mu \nu} F^{\alpha \beta} F_{\alpha \beta}\right)
$$

and $F_{\mu \nu}$ satisfies the Maxwell equations $F_{; \nu}^{\mu \nu}=0$. Using this Maxwell equation, we can derive

$$
F^{01}=\frac{Q}{r^{2}},
$$

where $Q$ is an integration constant playing the role of a electrostatic charge. Then the nonvanishing components of the Maxwell tensor $E^{\mu}{ }_{v}$ read as

$$
E_{v}^{\mu}=\frac{Q^{2}}{\kappa r^{4}} \operatorname{diag}(-1,-1,1,1) .
$$

In addition, $\mathcal{T}^{\mu}$ describes the energy-momentum tensor of surrounding field defined as [63]

$$
\begin{aligned}
& \mathcal{T}^{0}{ }_{0}=\mathcal{T}^{1}{ }_{1}=-\rho_{s}(r), \\
& \mathcal{T}^{2}{ }_{2}=\mathcal{T}^{3}{ }_{3}=\frac{1}{2}\left(1+3 \omega_{s}\right) \rho_{s}(r),
\end{aligned}
$$

where $\rho_{s}(r)$ is the energy density and satisfies barotropic equation of state $p_{s}=\omega_{s} \rho_{s}$ with the pressure $p_{s}(r)$ and state parameter $\omega_{s}$. The subscript " $s$ " denotes the surrounding field which can be a dust $\left(\omega_{d}=0\right)$, radiation $\left(\omega_{r}=1 / 3\right), \Lambda$ CDM type $\left(\omega_{\Lambda}=-1\right)$, quintessence and phantom field constructed by the combination of these fields.

From $H_{0}^{0}=T_{0}^{0}$ and $H_{2}^{2}=T_{2}^{2}$ components of Rastall field equations, the solution of four dimensional charged AdS black hole can be obtained as

$$
\begin{aligned}
& d s^{2}=-f(r) d t^{2}+\frac{1}{f(r)} d r^{2}+r^{2}\left(d \theta^{2}+\sin ^{2} \theta d \vartheta^{2}\right), \\
& f(r)=1-\frac{2 M}{r}+\frac{Q^{2}}{r^{2}}-\frac{N_{s}}{r^{\xi}}+\frac{r^{2}}{l_{\Lambda}^{2}}
\end{aligned}
$$

where $M$ and $N_{s}$ are two integration constants representing the black hole mass and surrounding field structure parameter, respectively. The parameters $\xi$ and $l_{\Lambda}$ are given by

$$
\begin{aligned}
\xi & =\frac{1+3 \omega_{s}-6 \psi\left(1+\omega_{s}\right)}{1-3 \psi\left(1+\omega_{s}\right)}, \\
\frac{1}{l_{\Lambda}^{2}} & =-\frac{\Lambda}{3(1-4 \psi)}, \quad \psi \equiv \kappa \lambda,
\end{aligned}
$$

where $l_{\Lambda}$ is the curvature radius, and $N_{s}$ is related to the energy density $\rho_{s}$

$$
\rho_{s}=-\frac{3 W_{s} N_{s}}{\kappa r^{\left(3\left(1+\omega_{s}\right)-12 \psi\left(1+\omega_{s}\right)\right) /\left(1-3 \psi\left(1+\omega_{s}\right)\right)}},
$$

where $W_{s}$ is

$$
W_{s}=-\frac{(1-4 \psi)\left(\psi\left(1+\omega_{s}\right)-\omega_{s}\right)}{\left(1-3 \psi\left(1+\omega_{s}\right)\right)^{2}} .
$$

Regarding the weak energy condition representing the positivity of any kind of energy density of the surrounding field, i.e., $\rho_{s} \geq 0$, imposes the following conditions

$$
W_{s} N_{s} \leq 0,
$$

which implies that for the surrounding field with $W_{s}>0$, we need $N_{s}<0$ and conversely for $W_{s}<0$, we need $N_{s}>0$. If $\omega_{s} \neq-1$, the constraint conditions for $\omega_{s}$ and $\xi$ can be derived, see Table 1. For the cosmological constant field $\omega_{s}=\omega_{\Lambda}=-1$, $\xi$ equals to -2 which is independent of $\psi$ and $W_{\Lambda}$ is $-1+4 \psi$. Then, we find

$$
N_{\Lambda}>0, \quad 0<\psi<\frac{1}{4}, \text { or } N_{\Lambda}<0, \quad \psi>\frac{1}{4}
$$

from the weak energy condition Equation (11). 
TABLE 2: Real root $r_{c}$ of Equation (23).

\begin{tabular}{|c|c|c|c|c|}
\hline \multirow{2}{*}{$r_{c}$} & \multicolumn{2}{|c|}{$\omega_{s}>-1$} & \multicolumn{2}{|c|}{$\omega_{s}<-1$} \\
\hline & $N_{s}>0$ & $N_{s}<0$ & $N_{s}>0$ & $N_{s}<0$ \\
\hline$\xi<-2$ & None & 1 & $0,1,2$ & None \\
\hline$-2<\xi<-1$ & 1 & None & None & $0,1,2$ \\
\hline$-1<\xi<0$ & $0,1,2$ & None & None & 1 \\
\hline $0<\xi \leq 2$ & \multicolumn{4}{|c|}{1} \\
\hline $2<\xi$ & None & 1 & $0,1,2$ & None \\
\hline
\end{tabular}

\section{Critical Behaviors of Charged AdS Black} Holes

In the extended phase space, we interpret the cosmological constant $\Lambda$ as a positive thermodynamic pressure $P$ in the geometric units $G_{N}=\hbar=c=1$

$$
P=-\frac{\Lambda}{8 \pi}=\frac{3(1-4 \psi)}{8 \pi l_{\Lambda}^{2}} \text {. }
$$

In terms of horizon radius $r_{+}$, mass $M$, Hawking temperature $T$, entropy $S$, and electromagnetic potential $\Phi$ of the charged AdS black holes can be written as

$$
\begin{aligned}
& M=\frac{1}{2}\left(\frac{Q^{2}}{r_{+}}+r_{+}-\frac{N_{s}}{r_{+}^{\xi-1}}+\frac{8 \pi P r_{+}^{3}}{3(1-4 \psi)}\right), \quad S=\pi r_{+}^{2}, \\
& T=\frac{1}{4 \pi}\left(\frac{1}{r_{+}}+\frac{8 \pi P r_{+}}{1-4 \psi}-\frac{Q^{2}}{r_{+}^{3}}+\frac{(\xi-1) N_{s}}{r_{+}^{1+\xi}}\right), \quad \Phi=\frac{Q}{r_{+}} .
\end{aligned}
$$

Here the black hole mass $M$ should be considered as the enthalpy rather than the internal energy of the gravitational system. Moreover, those thermodynamic quantities should satisfy first law of black hole thermodynamics

$$
d M=T d S+V d P+\Theta_{s} d N_{s}+\Phi d Q,
$$

where the thermodynamic volume $V$ conjugate to $P$ equals to $4 \pi r_{+}^{3} / 3(1-4 \psi)$ and $\Theta_{s}$ as a generalized force conjugate to the parameter $N_{s}$ reads as $-r_{+}^{1-\xi} / 2$.

Using Equation (17), the equation of state $P(V, T)$ can be written as

$$
P=\frac{(1-4 \psi)}{2 r_{+}}\left[T-\frac{1}{4 \pi r_{+}}+\frac{Q^{2}}{4 \pi r_{+}^{3}}-\frac{(\xi-1) N_{s}}{4 \pi r_{+}^{1+\xi}}\right]
$$

As usual, a critical point occurs when $P$ has an inflection point

$$
\left.\frac{\partial P}{\partial r_{+}}\right|_{T=T_{c}, r_{+}=r_{c}}=\left.\frac{\partial^{2} P}{\partial r_{+}^{2}}\right|_{T=T_{c}, r_{+}=r_{c}}=0,
$$

where the subscript " $c$ " denotes the values of the physical quantities at the critical point. When $\omega_{s} \neq-1$, we can obtain

$$
\begin{gathered}
T_{c}=\frac{\xi r_{c}^{2}-2 Q^{2}(\xi-2)}{2 \pi(1+\xi) r_{c}^{3}}, \\
P_{c}=\frac{\left(\xi r_{c}^{2}-3 Q^{2}(\xi-2)\right)\left(3 \omega_{s}-1\right)}{24 \pi r_{c}^{4}(\xi-2)\left(\omega_{s}+1\right)},
\end{gathered}
$$

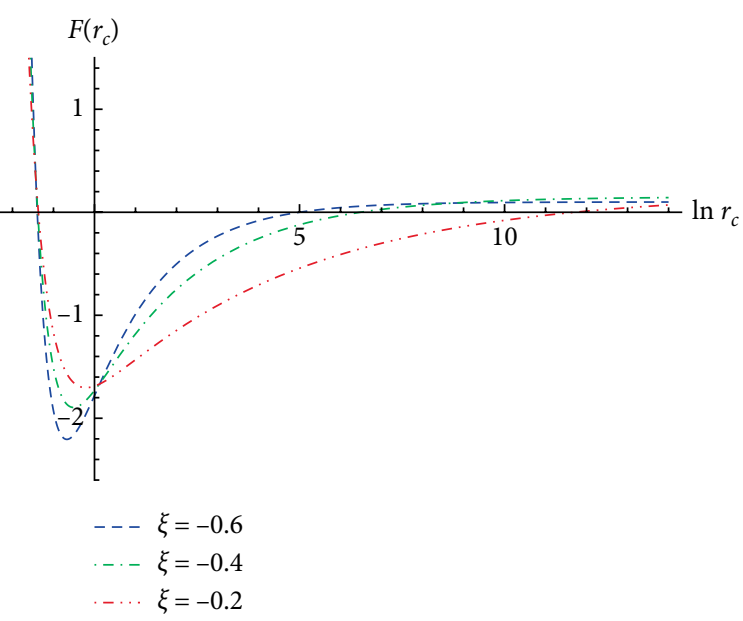

(a)

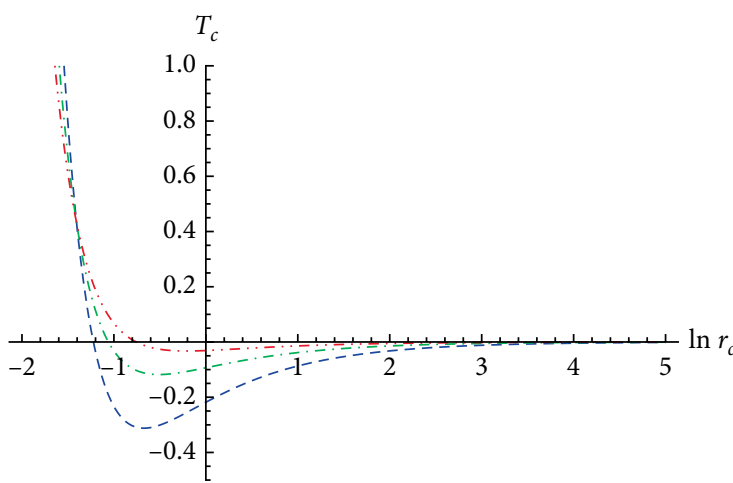

$$
\begin{aligned}
& ---\xi=-0.6 \\
& -.-\xi=-0.4 \\
& -\cdots \quad \xi=-0.2
\end{aligned}
$$

(b)

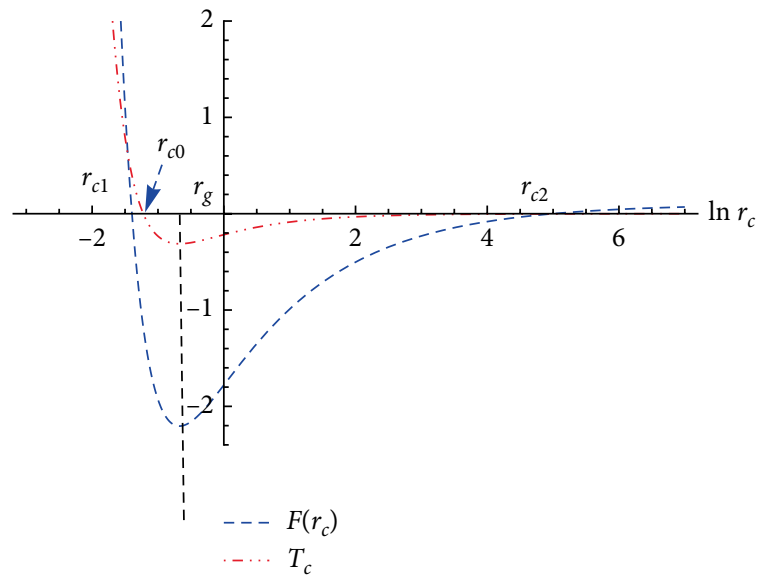

(c)

FIGURE 1: The $F\left(r_{c}\right)-\ln r_{c}$ and $T_{c}-\ln r_{c}$ diagrams with $Q=0.1$ and $N_{s}=0.1113$ for different values of $\xi$.

and the equation for critical horizon radius $r_{c}$

$$
F\left(r_{c}\right)=(1-\xi)(1+\xi)(2+\xi) N_{s}-2 r_{c}^{\xi-2}\left(r_{c}^{2}-6 Q^{2}\right)=0
$$


with Equation (11). Evidently, it is hard to derive any analytic solution for the critical quantities $r_{c}, T_{c}$, and $P_{c}$ from Equations (21)-(23). By analyzing the asymptotical behavior of function $F\left(r_{c}\right)$ at the horizon and infinity, we summarize the number of real root $r_{c}$ of Equation (23) in Table 2. The discussion for cosmological constant field $\omega_{s}=-1$ will be shown in the last part of this section.

Taking the region of $\omega_{s}>-1,-1<\xi<0$ and $N_{s}>0$ for instance, the function $F\left(r_{c}\right)$ [Equation (23)] approaches $+\infty$ near the origin $r \rightarrow 0$ and tends to $-N_{s}(1-\xi)(1+\xi)(2+\xi)(>0)$ when $r \rightarrow+\infty$. For the critical temperature $T_{c}$ [Equation (21)], it approaches $+\infty$ near the origin $r \rightarrow 0, T_{c}$ disappears at $r_{c}=r_{c 0}=\sqrt{2(\xi-2) / \xi} Q$ and tends to 0 when $r \rightarrow+\infty$, see Figures 1(a) and 1(b). On the other hand, the derivative of $F\left(r_{c}\right), T_{c}$, and $P_{c}$ leads to a same expression

$$
F^{\prime}\left(r_{c}\right)=T_{c}^{\prime}=P_{c}^{\prime}=-6 Q^{2}(-2+\xi)+r_{c}^{2} \xi,
$$

which vanishes at $r_{c} \equiv r_{g}=\sqrt{6(\xi-2) / \xi} Q$. From $F\left(r_{c}\right)=0$, the two real positive critical radius $r_{c 1}$ and $r_{c 2}$ (if exist) satisfy $r_{c 1}<r_{g}<r_{c 2}$. We have $T_{c}\left(r_{c 2}\right)<0$ because of $r_{c 0}<r_{g}<r_{c 2}$ and $T_{c}\left(r_{c 1}\right)^{g}>0$ if $r_{c 1}<r_{c 0}$, see Figure 1(c). In general, a black hole should possess positive temperature $T>0$, and reduces to extremal one when $T=0$. Moreover, holding positive temperature $T>0$ makes it possible to set up an analogy between the realistic Van der Waals phase transition for liquid/gas system [64] and SBH/LBH phase transition for the charged AdS black hole in the Rastall gravity. For the four dimensional ReissnerNordström AdS black hole, Kubiznak and Mann derived one set of positive critical quantities $r_{c}, P_{c}$, and $T_{c}$ by utilizing Equation (20) in the extended phase space, and further recovered that Reissner-Nordström AdS black hole admits VdWlike $\mathrm{SBH} / \mathrm{LBH}$ phase transition in the Einstein gravity [32].

Besides the VdW-like SBH/LBH phase transition, Ref. [48] firstly proposed the reentrant phase transition (RPT) for Born-Infeld AdS black hole in GR, if there exist more than one positive critical points. Nevertheless, the RPT does not happen in this region $\left(\omega_{s}>-1,-1<\xi<0\right.$ and $\left.N_{s}>0\right)$ for charged AdS black hole in Rastall gravity because of the unphysical critical temperature $T_{c}\left(r_{c 2}\right)<0$, even though there could exist two positive real critical radius $r_{c}$ from Table 2 .

In addition, to study the possible phase transitions in the system, we need to discuss the Gibbs free energy, which obeys the following thermodynamic relation $G=M-T S$ with

$$
G=\frac{r_{+}}{4}+\frac{3 Q^{2}}{4 r_{+}}-\frac{2 P \pi r_{+}^{3}}{3(1-4 \psi)}-\frac{(1+\xi) N_{s}}{4 r_{+}^{\xi-1}},
$$

where $r_{+}$is understood as a function of pressure and temperature, $r_{+}=r_{+}(P, T)$, via equation of state [Equation (19)]. In the rest of this section, we mainly focus on that regions possessing more than one real roots of $r_{c}$ from Table 2, and then consider the critical behaviors of charged AdS black holes surrounded by dust, radiation, quintessence, phantom, or $\Lambda$ CDM type fields.

3.1. Black Hole Surrounded by Dust or Quintessence Fields. For the dust surrounding field, we set $\omega_{d}=0$. Taking $\xi=-0.8$, $Q=0.1$ and $N_{d}=0.1$ for instance, one can obtain two critical radius $r_{c 1}=0.245$ and $r_{c 2}=120.762$, and corresponding critical pressure and temperature $P_{c 1} \approx 0.0463, T_{c 1} \approx 0.4104$ and $P_{c 2} \approx-2.5984 * 10^{-7}, T_{c 2} \approx-0.00527166$. So, there only exists one physical critical point, which has been verified in the above part. As shown in Figure 2, $P-r_{+}$diagram displays that the solid curve represents critical isotherm at $T=T_{c 1}$ and the dashed and dotted curves correspond to $T>T_{c 1}$ and $T<T_{c 1}$, respectively. In the $G-T$ diagram, the solid curve represents $P<P_{c 1}$, the dotted curve corresponds to $P>P_{c 1}$, and the dashed curve is for $P=P_{c 1}$. We observe standard swallowtail behavior. Moreover, the $P-T$ diagram shows the coexistence line of the first-order $\mathrm{SBH} / \mathrm{LBH}$ phase transition terminating at a critical point. These plots are analogous to typical behavior of the liquid-gas phase transition of the VdW fluid.

We turn to discuss the quintessence field. The quintessential dark energy is governed by an equation of state of the form $p_{q}=\omega_{q} \rho_{q}$ with the state parameter $\omega_{q} \in(-1,-1 / 3)[63]$. Here we choose a ordinary example $\omega_{q}=-2 / 3[63,65]$. Taking $\xi=-0.7, Q=0.4$ and $N_{q}=0.4$, one can obtain two critical radius $r_{c 1}=1.055$ and $r_{c 2}=17.85$, and corresponding critical pressure and temperature $P_{c 1} \approx 0.0184, T_{c 1} \approx 0.0382$ and $P_{c 2} \approx-0.000096, T_{c 2} \approx-0.0207$. Therefore, there only exists one physical critical point, which implies the appearance of VdW-like SBH/LBH phase transition. The $P-r_{+}, G-T$, and $P-T$ diagrams are similar with Figure 2, so we do not plot these figures in this case.

3.2. Black Hole Surrounded by Radiation Field. With regard to the case of $\omega_{r}=1 / 3$, one have $\xi=2$, which is independent of $\psi$ from Equation (11). Then, the black hole solution (10) becomes

$$
f(r)=1-\frac{2 M}{r}+\frac{Q^{2}-N_{r}}{r^{2}}+\frac{r^{2}}{l_{\Lambda}^{2}},
$$

Obviously, the parameter $N_{r}$ plays a shift role in the charge $Q$. It's worthy to point out that the condition $Q^{2}>N_{r}$ needs to be satisfied so that we can derive the possible phase transition. Then, the equation of state $P(V, T)$ reduces to

$$
P=\frac{(1-4 \psi)}{2 r_{+}}\left(T-\frac{1}{4 \pi r_{+}}+\frac{Q^{2}}{4 \pi r_{+}^{3}}-\frac{N_{r}}{4 \pi r_{+}^{3}}\right)
$$

According the judgement condition (20), corresponding critical values read as

$$
\begin{aligned}
& r_{c}=\sqrt{6\left(Q^{2}-N_{r}\right)}, \quad P_{c}=\frac{1-4 \psi}{96 \pi\left(Q^{2}-N_{r}\right)}, \\
& T_{c}=\frac{1}{3 \sqrt{6\left(Q^{2}-N_{r}\right)}}, \quad Q^{2}>N_{r}, \quad \psi<\frac{1}{4} .
\end{aligned}
$$

Then, VdW-like SBH/LBH phase transition occurs for charged AdS black hole in the region of $Q^{2}>N_{r}$ and $\psi<1 / 4$, which is similar as that for charged AdS black hole in Einstein gravity [32].

3.3. Black Hole Surrounded by Phantom Field. For the phantom field, it possesses the supernegative equation of state $\omega_{p}<-1$ [65]. Nevertheless, when a black hole immersed this phantom 


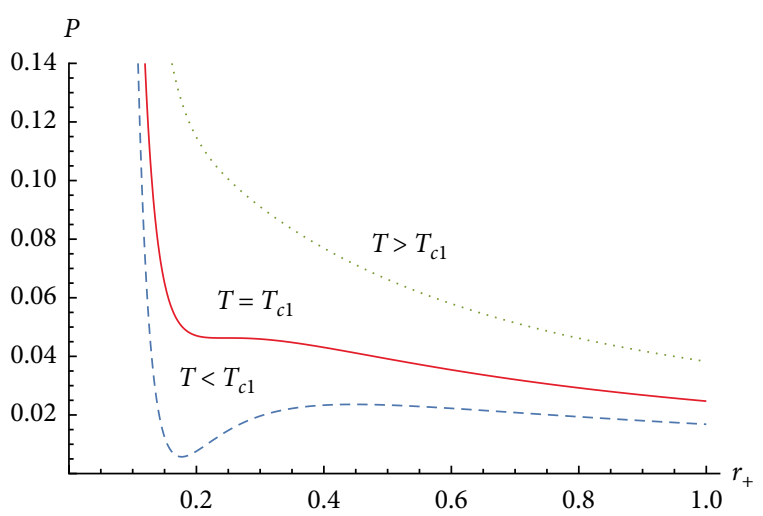

(a)

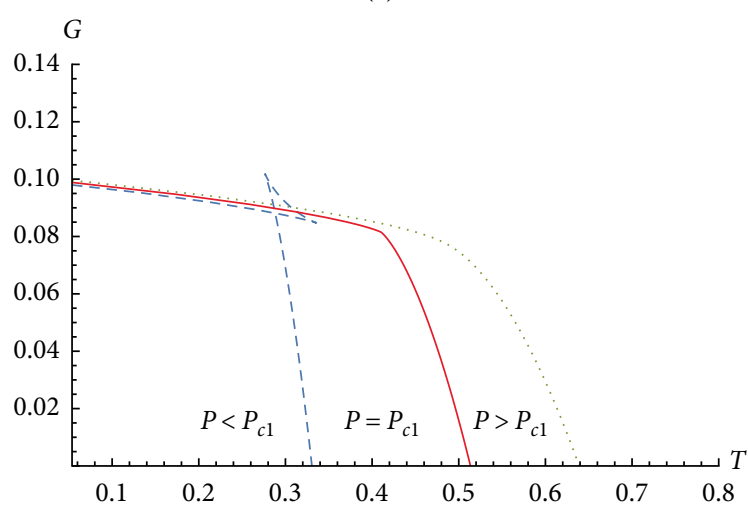

(b)

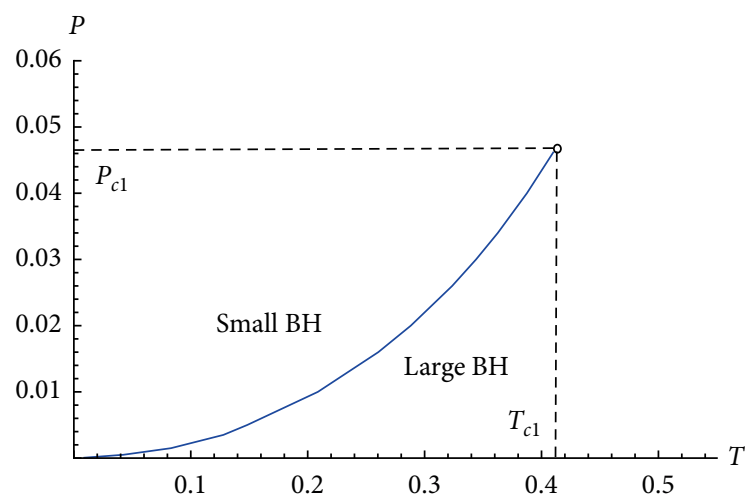

(c)

Figure 2: The $P-r_{+}, G-T$, and $P-T$ diagrams of fourdimensional charged AdS black holes with $\omega_{d}=0, \xi=-0.8, Q=0.1$ , and $N_{d}=0.1$. There is one critical point, which corresponds to VdW-like SBH/LBH phase transition when $T<T_{c 1} \approx 0.4104$ and $P<P_{c 1} \approx 0.0463$.

field, it gives rise to $p_{p}+\rho_{p}<0$ and $T_{p} s_{p}<0$, and leads to two alternatives: either the entropy density $s_{p}$ is negative with positive temperature $T_{p}$ or the entropy density $s_{p}$ is positive but the temperature is negative. With $T_{p}>0$, the entropy of black hole decreases as it accretes the phantom energy, meanwhile a decrease in the energy density of phantom field decreases the entropy of phantom field $[66,67]$. Finally, the total entropy of thermodynamic system consisting of phantom field entropy and black holes entropy will decrease simultaneously, which violates the generalized second law (GSL) of thermodynamics, and then the accretion process could never occur in this case.

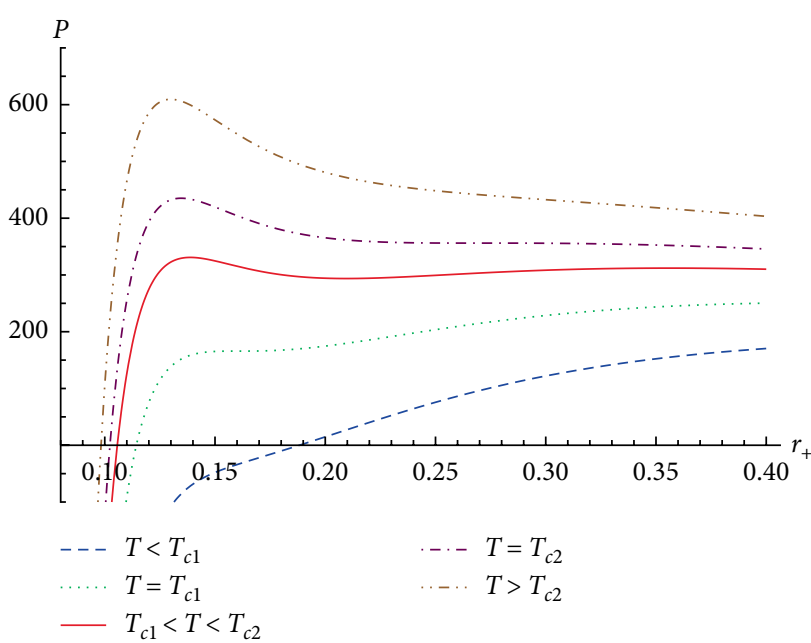

(a)

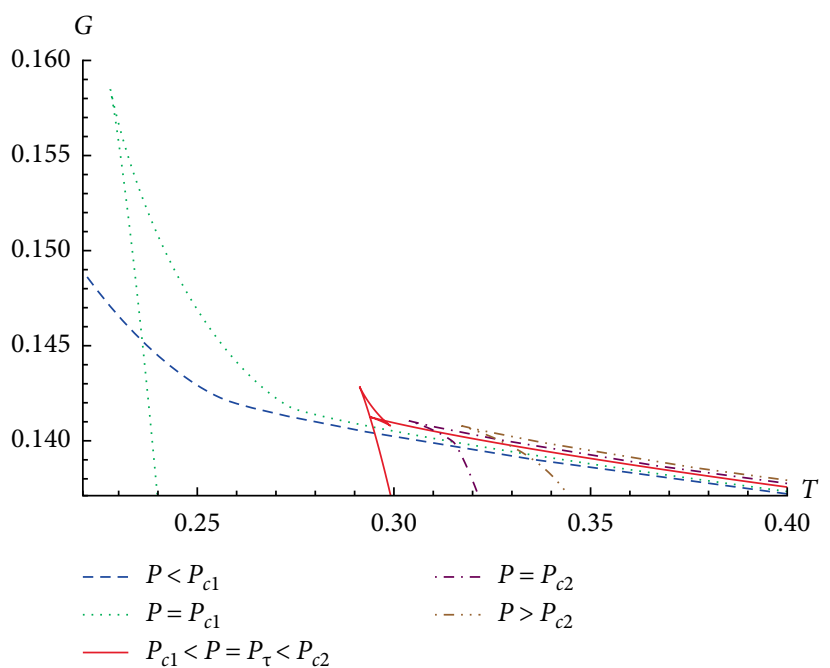

(b)

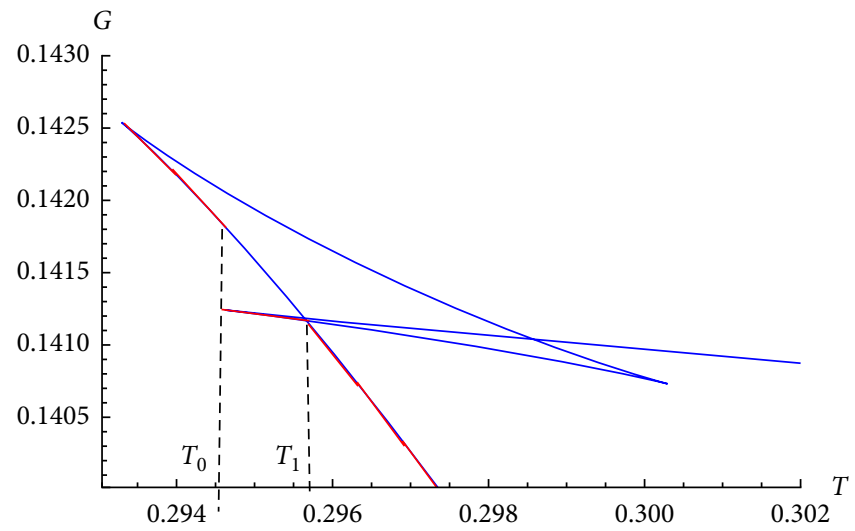

(c)

Figure 3: The $P-r_{+}$and $G-T$ diagrams of four-dimensional charged AdS black holes with $\omega_{p}=-1.03, \xi=2.1, Q=0.4$, and $N_{p}=0.1113$. It shows the existence of two critical points $\left(P_{c 1}=165.8340, T_{c 1}=0.2743\right)$ and $\left(P_{c 2}=356.10, T_{c 2}=0.3153\right)$. For $P \in\left(P_{\tau}, P_{z}\right)$, we observe a "zeroth-order phase transition" at $T_{0}$ signifying the onset of an RPT in (c).

In terms of $T_{p}<0$, the accretion of phantom field by this black hole is also not possible if the mass of black hole is above 


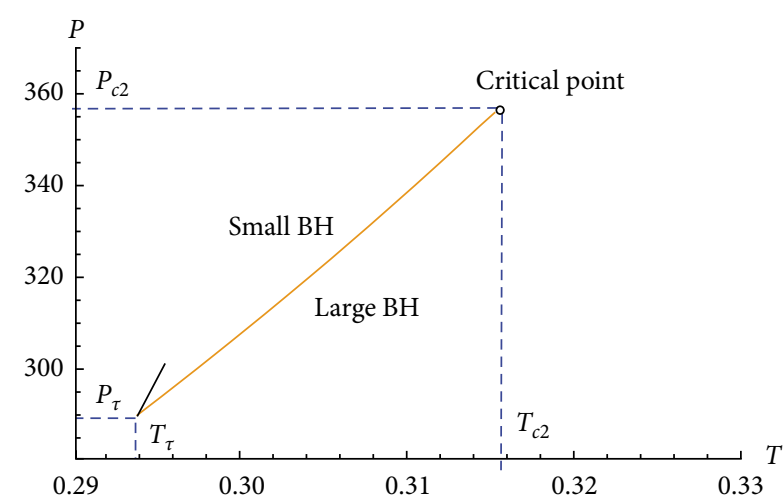

(a)

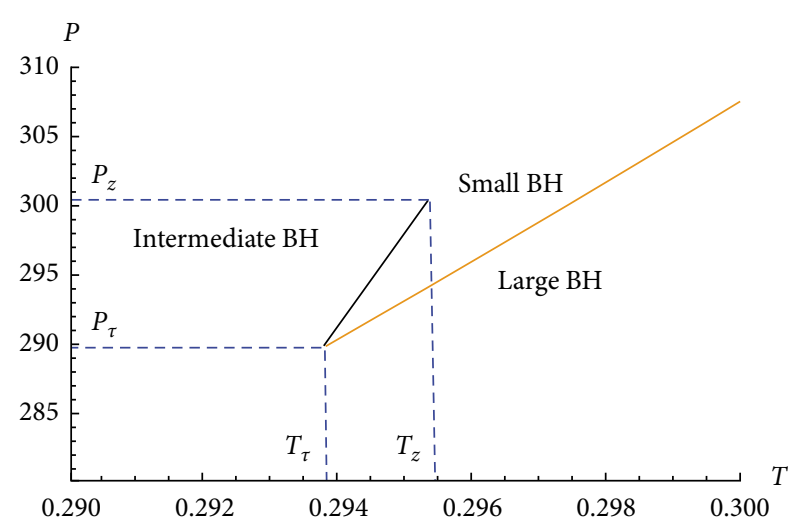

(b)

Figure 4: The $P-T$ diagram of four-dimensional charged AdS black holes with $\omega_{p}=-1.03, \xi=2.1, Q=0.4$, and $N_{p}=0.1113$. The thick solid line initiating from $\left(P_{c 2}, T_{c 2}\right)$ and terminates at $\left(P_{\tau}, T_{\tau}\right)$ corresponds to the co-existence line of VdW-like SBH/LBH phase transition. The solid line commencing from $\left(P_{z}, T_{z}\right)$ and terminates at $\left(P_{\tau}, T_{\tau}\right)$ denotes the coexistence line of small and intermediate black holes indicating the RPT.

from corresponding critical value [68-71]. Here we only discuss thermodynamics and phase transitions of charged AdS black holes in the Rastall gravity. Therefore, we can assume the phantom field with $T_{p}>0$ for simplicity, so that the thermodynamic system consisting of charged AdS black hole and phantom field is always stable.

Recently, Kumar and Xu adopted the observational data sets of SNLS3, BAO, Planck + WMAP9 + WiggleZ to determine the parameter $\omega_{p}=-1.06_{-0.13}^{+0.11}[72]$. Moreover, the Planck 2018 results recovers $\omega_{p}=-1.03 \pm 0.03$ [73]. In view of these constraints on $\omega_{p}$ from observations, we consider the phantom field with $\omega_{p}=-1.03$. With $N_{p}>0$ and $\xi>2$ in Table 2, the function $F\left(r_{c}\right)$ [Equation (23)] tends to $-N_{p}(\xi-1)(\xi+1)(\xi+2)(<0)$ as $r_{c} \rightarrow 0$ and $-\infty$ when $r_{c} \rightarrow-\infty$. Based on $F\left(r_{c}\right)=0$ and Equations (21) and (22), we can obtain two positive critical points when the parameters $Q, \xi$, and $N_{p}$ satisfy

$$
\frac{2(\sqrt{3(\xi-2) / \xi} Q)^{\xi}}{(\xi-2)(\xi-1)(\xi+1)}<N_{s}<\frac{4(\sqrt{6(\xi-2) / \xi} Q)^{\xi}}{(\xi-2)(\xi-1)(\xi+1)(\xi+2)} .
$$

By using the graphical and numerical method, $P-r_{+}$and $G-T$ diagrams of charged AdS black hole with $\xi=2.1$, $Q=0.4$, and $N_{p}=0.1113$ are displayed in Figure 3. One can find two physical critical points [Figure 3(a)]. For $P<P_{c 2}\left(T<T_{c 2}\right)$, the VdW-like SBH/LBH phase transition occurs, and terminates at $P=P_{\tau}\left(T=T_{\tau}\right)$. At the same time, there also exists a certain range of $P \in\left(P_{\tau}, P_{z}\right)$, namely $T \in\left(T_{\tau}, T_{z}\right)$, for which the global minimum of $G$ is discontinuous; see Figure 3(c). In this range of temperatures, two separate branches of intermediate size and small size black holes co-exist. They are separated by a finite jump in $G$, which is so-called "zeroth-order phase transition".

This novel situation can be also clearly illustrated in the $P-T$ diagrams in Figure 4. There is the expected SBH/LBH line of co-existence, which initiates from the critical point $\left(T_{\tau}, P_{\tau}\right)$ and terminates at $\left(T_{c 2}, P_{c 2}\right)$. Especially, a "triple point" between the small (SBH), intermediate (IBH), and large black holes $(\mathrm{LBH})$ emerges in the point $\left(T_{\tau}, P_{\tau}\right)$. In the region of $T \in\left(T_{\tau}, T_{z}\right)$, a new IBH/SBH line of coexistence appears and then it terminates in another critical point $\left(T_{z}, P_{z}\right)$. These values are obtained as

$$
\begin{aligned}
& \left(T_{\tau}, T_{z}, T_{c 2}\right) \approx(0.2938,0.2954,0.3153), \\
& \left(P_{\tau}, P_{z}, P_{c 2}\right) \approx(289.8545,300.2650,356.10) .
\end{aligned}
$$

Considering the other regions of $N_{p}>0, \xi<-2$, or $N_{p}<0,-2<\xi<-1$ in Table 2, two positive roots of $r_{c 1}$ and $r_{c 2}$ could appear from equation $F\left(r_{c}\right)=0$. Taking $N_{p}>0$ and $\xi<-2$ for instance, we choose $\omega_{p}=-1.03, \xi=-2.4, Q=0.2$, and $N_{p}=0.4$, and find that there only exists the VdW-like $\mathrm{SBH} / \mathrm{LBH}$ phase transition in the region of $T_{c 1}<T_{c}<T_{c 2}$ and $P_{c 1}<P_{c}<P_{c 2}$ with

$$
\left(P_{c 1}, P_{c 1}\right) \approx(0.17836,0.43678), \quad\left(T_{c 2}, T_{c 2}\right) \approx(0.23295,0.57949) \text {, }
$$

see Figure 5. Similar discussion can be done in the region of $N_{p}<0,-2<\xi<-1$ and $\omega_{p}=-1.03$, we find there only is the VdW-like SBH/LBH phase transition, even though there also exist two different physical critical points: $\left(r_{c 1}, T_{c 1}, P_{c 1}\right)>0$ and $\left(r_{c 2}, T_{c 2}, P_{c 2}\right)>0$.

3.4. Black Hole Surrounded $\Lambda C D M$ Type Field. In case of $\omega_{\Lambda}=-1$, namely $\xi=-2$, the critical horizon radius, temperature, and pressure are obtained as

$$
r_{c}=\sqrt{6} Q, \quad T_{c}=\frac{1}{3 \sqrt{6} \pi Q} \quad P_{c}=\frac{\left(1+36 N_{\Lambda} Q^{2}\right)(1-4 \psi)}{96 \pi Q^{2}} .
$$

Holding $P_{c}>0$, the parameters $N_{\Lambda}$ and $\psi$ should satisfy $N_{\Lambda}<-1 / 36 Q^{2}$ for $\psi>1 / 4$ and $N_{\Lambda}>0$ for $0<\psi<1 / 4$ in terms of the condition (15). Finally, VdW-like SBH/LBH phase transition occurs in these regions, which is similar as that for charged AdS black hole in Einstein gravity [32]. 


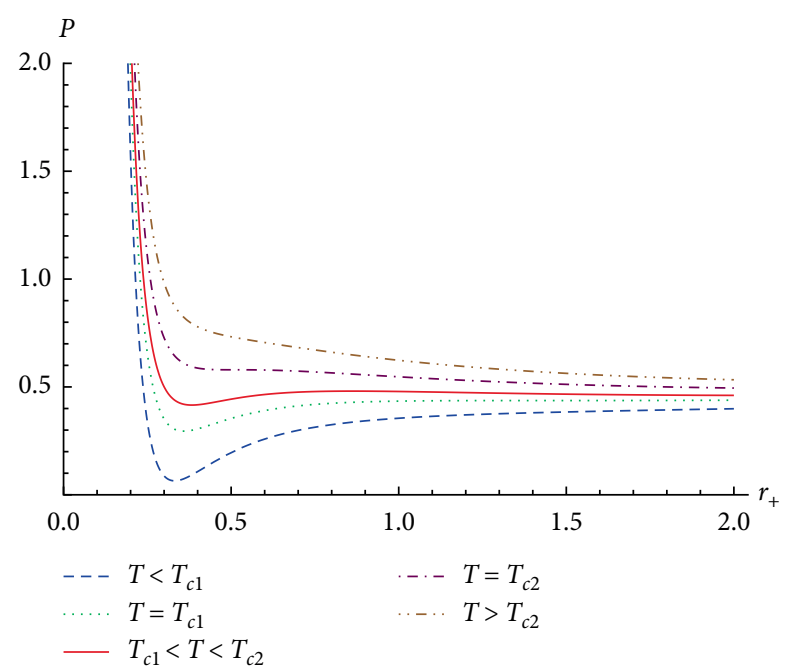

(a)

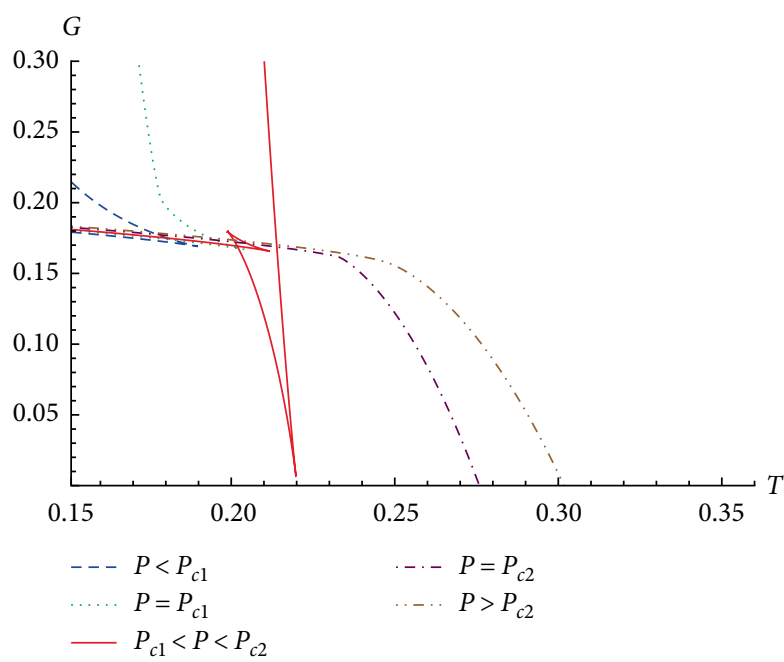

(b)

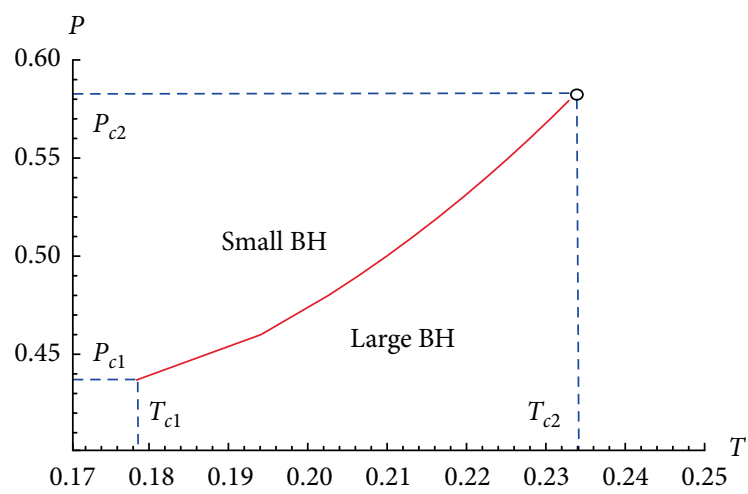

(c)

Figure 5: The $P-r_{+}, G-T$, and $P-T$ diagrams of fourdimensional charged AdS black holes with $\omega_{p}=-1.03, \xi=-2.4$, $\mathrm{Q}=0.2$, and $N_{p}=0.4$. The VdW-like SBH/LBH phase transition happens in the region of $T_{c 1}<T<T_{c 2}$ and $P_{c 1}<P<P_{c 2}$.

\section{Conclusion and Discussion}

We have obtained charged AdS black holes solutions surrounded by perfect fluid in the context of four dimensional
Rastall theory. Regarding the weak energy condition of energy density and asymptotic behavior of function $F\left(r_{c}\right)$, we derived the constraint conditions for the parameters $N_{s}, \omega_{s}$ and $\xi$ or $\psi$, as shown in Tables 1 and 2. Later, we chose some special surrounding fields which can be a dust, radiation, quintessence, $\Lambda$ CDM type and phantom field, and found that the Van der Waals like SBH/LBH phase transition happens for the charged AdS black holes with dust, radiation or quintessence and $\Lambda \mathrm{CDM}$ type fields. In particular, the charged AdS black hole surrounded by phantom field showed more interesting phenomenon in the region of $N_{p}>0$ and $\xi>2$. When the parameters $Q$, $\xi$, and $N_{p}$ satisfy $\left(2(\sqrt{3(\xi-2) / \xi} Q)^{\xi} /(\xi-2)(\xi-1)(\xi+1)\right)<N_{p}$ $<\left(4(\sqrt{6(\xi-2) / \xi} Q)^{\xi} /(\xi-2)(\xi-1)(\xi+1)(\xi+2)\right)$ and $\xi>2$, we obtained two positive critical points and so-called RPT phase transition occurs, besides the VdW-like $\mathrm{SBH} / \mathrm{LBH}$ phase transition. However, in the region of $N_{p}>0, \xi<-2$, or $N_{p}<0$, $-2<\xi<-1$, there only exist the VdW-like SBH/LBH phase transition, even though it has two physical critical points.

Comparing with the Maxwell field, the Born-Infeld AdS black hole, and charged AdS black hole with Yang-Mills field in the Einstein gravity have proposed more interesting thermodynamical properties. In the Rastall gravity, one can also construct new black holes with nonlinear electrodynamics, and then explore whether the Van der Waals or reentrant phase transition can appear.

\section{Data Availability}

No data were used to support this study.

\section{Conflicts of Interest}

The authors declare that they have no conflicts of interest.

\section{Acknowledgments}

This work is supported by the National Natural Science Foundation of China under Grant Nos. 11605152, 11675139, and 51802247, and Natural Science Foundation of Jiangsu Province (Grant No. BK20160452)

\section{Supplementary Materials}

The supplementary files are data for the Figures 2(b) and 2(c); Figures 3(b) and 3(c), Figure 4, and Figures 5(b) and 5(c). (Supplementary Materials)

\section{References}

[1] A. G. Riess, L.-G. Strolger, J. Tonry et al., “Type Ia supernova discoveries at $z>1$ from the hubble space telescope: evidence for past deceleration and constraints on dark energy evolution," The Astrophysical Journal, vol. 607, no. 2, p. 665, 2004.

[2] E. Komatsu, K. M. Smith, J. Dunkley et al., "Seven-year Wilkinson microwave anisotropy probe (wmap ${ }^{*}$ ) observations: 
cosmological interpretation," The Astrophysical Journal Supplement Series, vol. 192, no. 2, p. 18, 2011.

[3] P. J. E. Peebles and B. Ratra, "The cosmological constant and dark energy," Reviews of Modern Physics, vol. 75, p. 559, 2003.[astro-ph/0207347]

[4] E. J. Copeland, M. Sami, and S. Tsujikawa, "Dynamics of dark energy," International Journal of Modern Physics D, vol. 15, pp. 1753-1935, 2006.

[5] S. Wang, Y. Wang, and M. Li, "Holographic dark energy," Physics Report, vol. 696, p. 1, 2017, https://arxiv.org/abs/1612.00345.

[6] P. Rastall, "Generalization of the Einstein theory," Physical Review D, vol. 6, p. 3357, 1972.

[7] P. Rastall, "A theory of gravity," Canadian Journal of Physics, vol. 54, no. 1, pp. 66-75, 1976.

[8] H. Moradpour, Y. Heydarzade, F. Darabi, and I. G. Salako, "A generalization to the Rastall theory and cosmic eras," The European Physical Journal C, vol. 77, no. 4, p. 259, 2017, https:// arxiv.org/abs/1704.02458.

[9] H. Moradpour, "Thermodynamics of flat FLRW universe in Rastall theory," Physics Letter B, vol. 757, pp. 187-191, 2016.

[10] I. G. Salako, M. J. S. Houndjo, and A. Jawad, "Generalized Mattig's relation in Brans-Dicke-Rastall gravity," International Journal of Modern Physics D, vol. 25, no. 7, 2016.

[11] C. E. M. Batista, J. C. Fabris, O. F. Piattella, and A. M. VelasquezToribio, "Observational constraints on Rastall's cosmology," European Physical Journal C, vol. 73, p. 2425, 2013.

[12] Y. Heydarzade, H. Moradpour, and F. Darabi, "Black hole solutions in Rastall theory," Canadian Journal of Physics, vol. 95, no. 12, pp. 1253-1256, 2017, https://arxiv.org/abs/1610.03881.

[13] S. Chen, B. Wang, and R. Su, "Hawking radiation in a $d$ dimensional static spherically symmetric black hole surrounded by quintessence," Physical Review D, vol. 77, no. 12-15, 2008.

[14] Y. Heydarzade and F. Darabi, "Black hole solutions surrounded by perfect fluid in Rastall theory," Physics Letter B, vol. 771, pp. 365-373, 2017.

[15] K. Lin and W. L. Qian, "Neutral regular black hole solution in generalized Rastall gravity," Chinese Physics C, vol. 43, no. 8, 2019, https://arxiv.org/abs/1812.10100.

[16] K. Lin, Y. Liu, and W. L. Qian, "Higher dimensional powerMaxwell charged black holes in Einstein and Rastall gravity," General Relativity and Gravitation, vol. 51, no. 5, p. 62, 2019.

[17] R. Kumar and S. G. Ghosh, "Rotating black hole in Rastall theory," The European Physical Journal C, vol. 78, no. 9, p. 750, 2018, https://arxiv.org/abs/1711.08256.

[18] Z. Xu, X. Hou, X. Gong, and J. Wang, "Kerr-Newman-AdS black hole surrounded by perfect fluid matter in Rastall gravity," The European Physical Journal C, vol. 78, no. 6, p. 513, 2018.

[19] H. Moradpour and I. G. Salako, "Thermodynamic analysis of the static spherically symmetric field equations in Rastall theory," Advances in High Energy Physics, vol. 2016, Article ID 3492796, 5 pages, 2016, https://arxiv.org/abs/1606.06589.

[20] I. P. Lobo, H. Moradpour, J. P. Morais Graca, and I. G. Salako, "Thermodynamics of black holes in Rastall gravity," International Journal of Modern Physics, vol. 27, no. 7, Article ID 1850069, 2018.

[21] K. Bamba, A. Jawad, S. Rafique, and H. Moradpour, "Thermodynamics in Rastall gravity with entropy corrections," The European Physical Journal C, vol. 78, no. 12, p. 986, 2018, https://arxiv.org/pdf/1812.00753.
[22] S. Soroushfar, R. Saffari, and S. Upadhyay, "Thermodynamic geometry of a black hole surrounded by perfect fluid in Rastall theory," General Relativity and Gravitation, vol. 51, p. 130, 2019, https://arxiv.org/abs/1908.02133.

[23] J. P. Morais Graca and I. P. Lobo, "Scalar QNMs for higher dimensional black holes surrounded by quintessence in Rastall gravity," The European Physical Journal C, vol. 78, no. 2, p. 101, 2018.

[24] J. Liang, "Quasinormal modes of the schwarzschild black hole surrounded by the quintessence field in Rastall gravity ${ }^{*}$," Communication in Theorectical Physics, vol. 70, no. 6, p. 695, 2018.

[25] J. M. Maldacena, "The large $N$ limit of superconformal field theories and supergravity," Advances in Theoretical and Mathematical Physics, vol. 2, pp. 231-252, 1998, https://arxiv. org/abs/hep-th/9711200.

[26] S. S. Gubser, I. R. Klebanov, and A. M. Polyakov, "Gauge theory correlators from non-critical string theory," Physics Letter B, vol. 428, p. 105, 1998, https://arxiv.org/abs/hep-th/9802109.

[27] E. Witten, "Anti de sitter space and holography," Advances in Theoretical and Mathematical Physics, vol. 2, p. 253, 1998, https://arxiv.org/abs/hep-th/9802150.

[28] S. W. Hawking and D. N. Page, "Thermodynamics of black holes in anti-de sitter space," Communications in Mathematical Physics, vol. 87, no. 4, pp. 577-588, 1983.

[29] E. Witten, "Anti-de sitter space, thermal phase transition, and confinement In gauge theories," Advances in Theoretical and Mathematical Physics, vol. 2, p. 505, 1998, https://arxiv.org/abs/ hep-th/9803131.

[30] B. P. Dolan, "Pressure and volume in the first law of black hole thermodynamics," Classical and Quantum Gravity, vol. 28, 2011, https://arxiv.org/abs/1106.6260.

[31] B. P. Dolan, "The cosmological constant and the black hole equation of state," Classical and Quantum Gravity, vol. 28, 2011, https://arxiv.org/abs/1008.5023.

[32] D. Kubiznak and R. B. Mann, " $P-V$ criticality of charged AdS black holes," Journal of High Energy Physics, vol. 1207, p. 33, 2012, https://arxiv.org/abs/1205.0559.

[33] R. Zhao, H.-H. Zhao, M.-S. Ma, and L.-C. Zhang, "On the critical phenomena and thermodynamics of charged topological dilaton AdS black holes," The European Physical Journal C, vol. 73, p. 2645, 2013, https://arxiv.org/abs/1305.3725.

[34] D. C. Zou, S. J. Zhang, and B. Wang, "Critical behavior of Born-Infeld AdS black holes in the extended phase space thermodynamics," Physical Review D, vol. 89, 2014, https:// arxiv.org/abs/1311.7299.

[35] D. C. Zou, Y. Liu, and B. Wang, "Critical behavior of charged Gauss-Bonnet AdS black holes in the grand canonical ensemble," Physical Review D, vol. 90, no. 4, 2014, https://arxiv. org/abs/1404.5194.

[36] R. G. Cai, L. M. Cao, L. Li, and R. Q. Yang, " $P-V$ criticality in the extended phase space of Gauss-Bonnet black holes in AdS space," Journal of High Energy Physics, vol. 2013, no. 9, p. 5, 2013, https://arxiv.org/abs/1306.6233.

[37] M. H. Dehghani, S. Kamrani, and A. Sheykhi, " $P$ - $V$ criticality of charged dilatonic black holes," Physical Review D, vol. 90, 2014, https://arxiv.org/abs/1505.02386.

[38] J.-X. Mo and W.-B. Liu, " $P-V$ criticality of topological black holes in Lovelock-Born-Infeld gravity," European Physical Journal C, vol. 74, p. 2836, 2014, https://arxiv.org/abs/1401.0785. 
[39] M. Chabab, H. El Moumni, S. Iraoui, and K. Masmar, "Phase transitions and geothermodynamics of black holes in dRGT massive gravity," The European Physical Journal C, vol. 79, no. 4, p. 342, 2019.

[40] K. Bhattacharya and B. R. Majhi, "Thermogeometric study of van der Waals like phase transition in black holes: an alternative approach," https://arxiv.org/abs/1903.10370.

[41] A. Haldar and R. Biswas, "Geometrothermodynamic analysis and $P$ - $V$ criticality of higher dimensional charged Gauss-Bonnet black holes with first order entropy correction," General Relativity and Gravitation, vol. 51, no. 2, p. 35, 2019.

[42] H. Yazdikarimi, A. Sheykhi, and Z. Dayyani, "Critical behavior of Gauss-Bonnet black holes via an alternative phase space," Physical Review D, 2019, https://arxiv.org/abs/1903.09020.

[43] W. Xu, C. y. Wang, and B. Zhu, "Effects of Gauss-Bonnet term on the phase transition of a Reissner-Nordström-AdS black hole in $(3+1)$ dimensions," Physical Review D, vol. 99, no. 4, 2019.

[44] Y. P. Hu, H. A. Zeng, Z. M. Jiang, and H. Zhang, " $P$ - V criticality in the extended phase space of black holes in EinsteinHorndeski gravity," Physical Review D, 2009, https://arxiv.org/ abs/1812.09938.

[45] M. Zhang and R. H. Yue, "Phase transition and quasinormal modes for spherical black holes in 5D Gauss-Bonnet gravity," Chinese Physics Letters, vol. 35, no. 4, 2018.

[46] A. Dehyadegari, B. R. Majhi, A. Sheykhi, and A. Montakhab, "Universality class of alternative phase space and Van der Waals criticality," Physics Letter B, vol. 791, pp. 30-35, 2019, https://arxiv.org/abs/1811.12308.

[47] M. Jamil, B. Pourhassan, A. ovgun, and I. Sakalli, "PV criticality of Achucarro-Ortiz black hole in the presence of higher order quantum and GUP corrections," Gunasekaran:2012dq, Hendi:2012um, Zhao:2013oza, https://arxiv.org/abs/1811.02193

[48] S. Gunasekaran, R. B. Mann, and D. Kubiznak, "Extended phase space thermodynamics for charged and rotating black holes and Born-Infeld vacuum polarization," Journal of High Energy Physics, vol. 2012, no. 11, p. 110, 2012, https://arxiv.org/ abs/1208.6251.

[49] S. H. Hendi and M. H. Vahidinia, "Extended phase space thermodynamics and $P-V$ criticality of black holes with nonlinear source," Physical Review D, vol. 88, 2013, https://arxiv. org/abs/1212.6128.

[50] R. A. Hennigar, W. G. Brenna, and R. B. Mann, " $P-V$ criticality in quasitopological gravity," Journal of High Energy Physics, vol. 2015, no. 7, p. 077, 2015.

[51] M. Zhang, D. C. Zou, and R. H. Yue, "Reentrant phase transitions and triple points of topological AdS black holes in BornInfeld-massive gravity," Advances in High Energy Physics, vol. 2017, Article ID 3819246, 2017, https://arxiv.org/abs/1707.04101.

[52] A. övgün, " $P-V$ criticality of a specific black hole in $\mathrm{f}(\mathrm{R})$ gravity coupled with Yang-Mills field," Advances in High Energy Physics, vol. 2018, Article ID 8153721, 2018, https://arxiv.org/ abs/1710.06795.

[53] P. Cheng, S.-W. Wei, and Y.-X. Liu, "Critical phenomena in the extended phase space of Kerr-Newman-AdS black holes," Physical Review D, vol. 94, no. 2, 2016, https://arxiv.org/ abs/1603.08694.

[54] J. X. Mo, G.-Q. Li, and X.-B. Xu, "Combined effects of $f(R)$ gravity and conformally invariant Maxwell field on the extended phase space thermodynamics of higher-dimensional black holes," The European Physical Journal C, vol. 76, p. 545, 2016.
[55] M. S. Ma, L. C. Zhang, H. H. Zhao, and R. Zhao, "Phase transition of the higher dimensional charged Gauss-Bonnet black hole in de sitter spacetime," Advances in High Energy Physics, vol. 2015, Article ID 134815, 2015.

[56] J. X. Mo and W. B. Liu, " $P-V$ criticality of conformal anomaly corrected AdS black holes," Advances in High Energy Physics, vol. 2015, Article ID 206963, 2015.

[57] H. Xu, W. Xu, and L. Zhao, "Extended phase space thermodynamics for third order Lovelock black holes in diverse dimensions," The European Physical Journal C, vol. 74, p. 3074, 2014, https://arxiv.org/abs/1405.4143.

[58] W. Xu, H. Xu, and L. Zhao, "Gauss-Bonnet coupling constant as a free thermodynamical variable and the associated criticality," The European Physical Journal C, vol. 74, p. 2970, 2014.

[59] A. M. Frassino, D. Kubiznak, R. B. Mann, and F. Simovic, "Multiple reentrant phase transitions and triple points in Lovelock thermodynamics," Journal of High Energy Physics, vol. 2014, no. 9, 2014, https://arxiv.org/abs/1406.7015.

[60] S. W. Wei and Y. X. Liu, "Triple points and phase diagrams in the extended phase space of charged Gauss-Bonnet black holes in AdS space," Physical Review D, vol. 90, 2014, 044057.

[61] N. Altamirano, D. Kubiznak, R. B. Mann, and Z. Sherkatghanad, "Thermodynamics of rotating black holes and black rings: phase transitions and thermodynamic volume," Galaxies, vol. 2, p. 89, 2014, https://arxiv.org/abs/1401.2586.

[62] M. S. Ali, "Ehrenfest scheme for $P-V$ criticality of the $d$ dimensional-AdS black holes surrounded by perfect fluid in Rastall theory," https://arxiv.org/abs/1901.04318.

[63] V. V. Kiselev, "Quintessence and black holes," Classical and Quantum Gravity, vol. 20, pp. 1187-1198, 2003.

[64] N. Goldenfeld, Lectures on Phase Transitions and the Renormalization Group, Westview Press, New York, 1992.

[65] A. Vikman, “Can dark energy evolve to the phantom?," Physical Review D, vol. 71, Article ID 023515, 2005.

[66] J. A. de Freitas Pacheco and J. E. Horvath, "Generalized second law and phantom cosmology: accreting black holes," Classical and Quantum Gravity, vol. 24, pp. 5427-5434, 2007.

[67] J. A. de Freitas Pacheco, "Do soft gamma repeaters emit gravitational waves?" 1998, https://arxiv.org/abs/astro$\mathrm{ph} / 9805321$.

[68] J. A. de Freitas Pacheco, "Relativistic accretion into a ReissnerNordström black hole revisited," 2011, https://arxiv.org/ abs/1109.6798.

[69] M. Sharif and S. Iftikhar, "Accretion onto a charged higherdimensional black hole," The European Physical Journal C, vol. 76, no. 3, p. 147, 2016.

[70] A. K. Ahmed, U. Camci, and M. Jamil, "Accretion on ReissnerNordstrom-(anti)-de Sitter black hole with global monopole," Classical and Quantum Gravity, vol. 33, no. 21, p. 215012, 2016.

[71] A. R. Amani and H. Farahani, "Phantom accretion onto the Schwarzschild AdS black hole with topological defect," International Journal of Theoretical Physics, vol. 51, p. 2943, 2012.

[72] S. Kumar and L. Xu, "Observational constraints on variable equation of state parameters of dark matter and dark energy after Planck," Physics Letters B, vol. 737, pp. 244-247, 2014.

[73] Planck Collaboration, "Planck 2018 results. VI. Cosmological parameters,” 2018, https://arxiv.org/abs/1807.06209. 